\title{
Aspergillus flavus Brain Abscess in Immunocompetent Teenagers: A Case Series with Review of Literature
}

\author{
Mohammed Bangash \\ Department of Surgery, Faculty of Medicine, King Abdulaziz University, Jeddah 21589, Saudi Arabia.
}

\section{Corresponding Author:}

Dr. Mohammed Bangash

Email: mhbangash@hotmail.com

This is an Open Access article distributed under the terms of the Creative Commons Attribution License (creativecommons.org/ licenses/by/3.0).

Received : August 18, 2016

Accepted : November 24, 2016

Published : January 10, 2017

\begin{abstract}
Background: Aspergillus is a saprophytic filamentous fungus that is the predominant mold pathogen of the immunosuppressed population. Cerebral involvement with aspergilloma carries a very high mortality rate. Case Report: This article reports two cases of immunocompetent teenager females involved with cerebral aspergilloma with different outcomes. First patient was 15 year old girl who presented with headache after functional endoscopic surgery. CT scan showed subarachnoid hemorrhage with basilar tip aneurysm and hydrocephalus. Second patient was 19 years old female, presenting with generalized tonic-clonic seizure, CT brain showed right parietal mass. Aspergillus flavus was isolated from both patients. Conclusion: Aspergillosis is a rare presentation in immunocompetent patients with incidental diagnosis.
\end{abstract}

Keywords: Aneurysm, Aspergillosus, Aspergillus flavus, Headache, Mycetoma, Saudi Arabia.

\section{Introduction}

Aspergillus is a saprophytic filamentous fungus seen in immunosuppressed population [1], acquired through inhalation of spores [2]. Extension into the brain via hematologic or directly via paranasal sinuses carries a poor prognosis [3,4]. Apart from immunocompromised patients central nervous system aspergillosis is also seen in cardiac, renal, diabetic, organ transplantation and neutropenic patients [5-7]. The treatment of cerebral aspergilloma may include medical antifungal therapy and surgical debridment [1]. Aspergilloma in the brain is a rare disease in immunocompetent individuals. The previously reported cases were in elderly [8], two adult males [9,10], and an infant [11]. This article reports two cases of immunocompetent teenager females having cerebral aspergilloma with different outcomes.

\section{Case Reports}

\section{Case 1}

A 15 year old Chadian lady, not known of any previous medical illness, presented to Ear, Nose and Throat service complaining of 8 months history of frontal headache. The CT of paranasal sinuses showed left maxillary and ethmoid sinus fungal infection. The patient was taken to the Operating Room for functional endoscopic sinus surgery (FESS). Few hours after the surgery, the patient started complaining of severe headache that got worse next day with no response to analgesics. His Glascow coma scale (GCS) was 14. CT brain showed subarachnoid hemorrhage with evidence of hydrocephalus [Fig.1a].

External ventricular drain was inserted by the neurosurgeon and a CT angiogram showed 
basilar tip aneurysm [Fig.1b]. The patient was started on amphotericin B and dexamethasone. She was seronegative for HIV infection. The neurointerventional radiology service was not available at that time. The patient's level of consciousness deteriorated rapidly with GCS of 7. A repeated CT brain showed evidence of re-bleeding with pending herniation. The patient was taken to the operating room and a pterional craniotomy was done and through a transsylvian approach the aneurysm was clipped [Fig.2]. The dissection through the arachnoid plain was extremely difficult as the fungal tissues were invading the whole skull base. These fungal tissues had to be resected to identify the blood vessels and cranial nerves. The basilar, posterior communicating and posterior cerebral arteries looked thrombosed. The basilar tip was located above the clivus bone and was edematous and fragile. The histopathological report revealed non-necrotizing granuloma with fungal hyphae that was consistent with aspergillosis infection. The fungal culture grew Aspergillus flavus. After the surgery the patient's level of consciousness failed to improve. On $2^{\text {nd }}$ post-operative day, CT perfusion showed no evidence of cerebral perfusion. The patient passed away a day later.

\section{Case 2}

A 19 year old Saudi female university student, not known to have any previous medical illness, fell down suddenly in the classroom and had generalized tonic clonic convulsions. She was brought to the emergency department in a postictal state. She was given lorazepam and loaded with intravenous phenytoin. After a few hours she regained her consciousness fully with GCS of 15 , but having left homonomous upper quadrinopsia as only neurological deficit.

MRI brain showed right parietal mass measuring around $3.5 \times 2.5 \times 3.3 \mathrm{~cm}$ in size, intraaxial, solid exhibiting low signal intensity in almost all pulse sequences including the long TR and FLAIR sequences with wall of irregular lobular
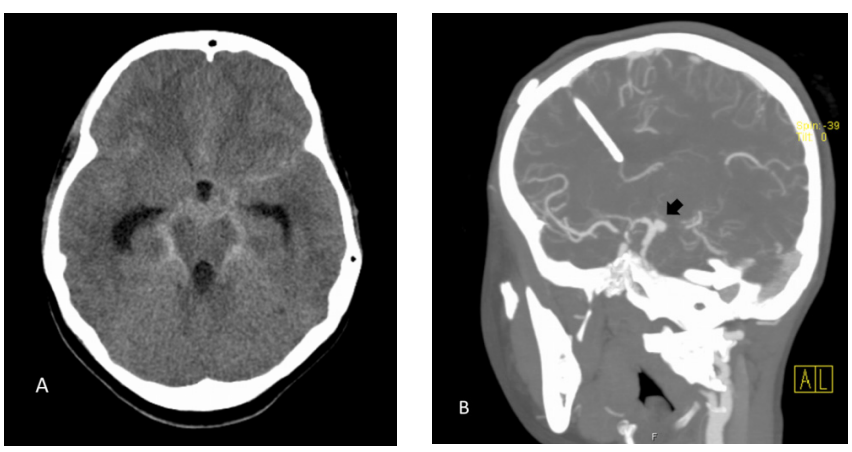

Fig.1(a): Non-enhanced CT brain shows basal cistern subarachnoid hemorrhage with evidence of hydrocephalus in term of dilated temporal horns, third and fourth ventricles. (b): CT angiogram shows the basilar tip aneurysm (black arrow) and the external ventricular drain in the lateral ventricle.

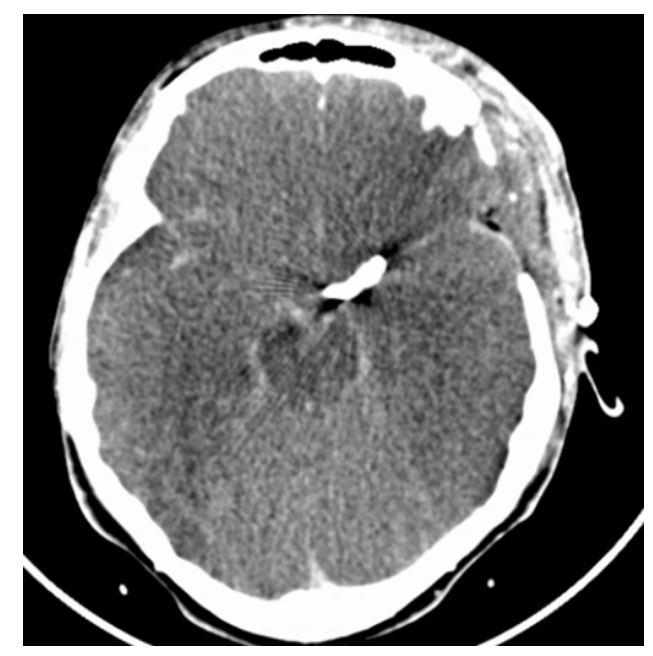

Fig.2: Post left pterional craniotomy CT brain shows the clip of the aneurysm through the transsylvian approach.

outlines and significant surrounding vasogenic edema resulting in effacement of the posterior horn and body of the right lateral ventricle. MR spectroscopy revealed high choline peak [Fig.3a]. The paranasal sinuses were free from any radiological abnormality. White blood cell count was $10.4 \mathrm{~K} / \mu \mathrm{L}$ with basophils of $0.2 \mathrm{~K} / \mu \mathrm{L}$. She was seronegative for HIV infection.

The patient underwent a craniotomy for resection of the intra-axial right parietal mass. 
The mass was hard in consistency with occasional calcifications, a cavetron aspirator was used to resect this mass. The histopathological report showed septate fungal hyphae with perivascular cuffing of inflammatory lymphocytes consistent with Aspergillosis. The fungal culture grew Aspergillus flavus. The patient was given an 18 months course of liposomal amphotericin B. The lesion disappeared completely from the MRI [Fig.3b], and the patient continued to have seizure that was easily controlled with a monotherapy (levetiracetam).

\section{Discussion}

Extrapulmonary aspergillus infection seen in $40 \%$ of cases, commonly presents as sinusitis and brain abscess [12]. Skull base aspergilloma is a dangerous and rare disease. Aspergillus commonly infects immunocompromised patients, with untreated central nervous system aspergillosis having 90\% mortality. Systemic anti-fungal therapy is mandatory in preventing subsequent vasculitis or meningoencephalitis. Some clinical trials have indicated superiority of voriconazole in comparison to amphotericin-B for first-line therapy in patients with Aspergillosis in terms of survival, response rates and safety $[13,14]$. For the second case the voriconazole was not available, so liposomal amphotericin B was used. In Middle east, Asia and Africa the sinuo-orbital, cerebral aspergillosis, aspergillus endophthalmitis are emerging diseases. The high frequency of A. flavus isolation from these patients may be due to higher load of the fungus in the environment such as in Saudi Arabia with hot weather [15]. Cerebral aneurysm induced by aspergilloma is rare and fatal [16-18].

CT of the brain may show a mass lesion with an irregular hypoattenuated center and surrounding brain edema with small calcifications within the mass. The MR brain may show on T2weighted images a heterogeneous hyperintense
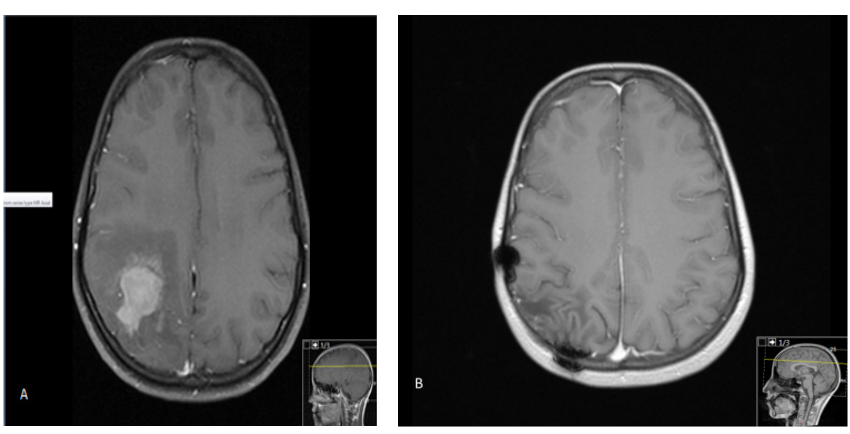

Fig.3(a): T1WI MRI with contrast showed right parietal intra-axial homogeneously enhancing mass with surrounding edema. (b): six years follow up MRI with complete resolution of the Aspergilloma.

core and a thick hypointense perimeter. Margins of the lesion can be well defined but irregular. On T1weighted images, the mass can be heterogeneous with occasional isointensity. A thick enhancing rim with a central non-enhancing portion is classic on postcontrast T1-weighted images. Perilesional vasogenic edema can be expected [10].

No definite answer is available to question how an immunocompetent individual got infected with such an opportunistic organism, however, the nutritional status, the environmental load with the organism and the duration of the exposure may play a role, thus, further studies and investigations are needed. Aspergillus species secrete different necrotizing factors that damage the neural cells which may contribute to brain lesions in patients with cerebral aspergillosis and, therefore, poor prognosis [19]. Pre-neurosurgical procedure load with steroid and anti-fungal treatment, such as posaconazole delayed-release, may help in avoiding the devastating effect of the necrotizing factors that damages the neural cells [20].

Two types of cranial aspergilloma are known. Type 1 involves the convexity and type 2 that involves the skull base (basal). Type 1 needs a craniotomy for complete excision of the fugal mass followed by long-term antifungal treatment with follow-up MRI at least biannually. Type 2 needs 
a careful evaluation of the cranial vasculature prior to any surgical intervention, in addition to the load with steroid and antifungal treatment preoperatively. The endovascular treatment can be considered in type 2 if an aneurysm is evident. After securing any present aneurysm, anticoagulant therapy may be added if thrombosis is an issue during the catheter angiogram.

Ozcelik et al. reported a successful control of cerebral aspergilloma with combination of surgery with intra-cavitary installation of the antifungal medication [21]. As this case looks like type 1 on the proposed classification, a radical surgery with systemic antifungal treatment may suffice to control the disease, however, intracavitary antifungal therapy is still a valid option of treatment.

\section{Conclusion}

Cranial aspirgilloma needs a team approach. The potential mortality has to be in mind and need to be mentioned to the patient and the next of kin early on. Early preoperative load with antifungal and steroid therapy may reduce the toxicity effect on neural tissues. Aggressive surgical debridement can help the patient.

Contributors: $\mathrm{MB}$ wrote the manuscript and was involved in patient management.

Funding: Faculty of Medicine at King Abdulaziz University; Competing interests: None stated.

\section{References}

1. Maschmeyer G, Haas A, Cornely OA. Invasive aspergillosis: epidemiology, diagnosis and management in immunocompromised patients. Drugs 2007;67(11):1567-1601.

2. Filler SG, Sheppard DC. Fungal invasion of normally nonphagocytic host cells. PLoS Pathog. 2006;2(12):e129.

3. Tekaia F, Latge JP. Aspergillus fumigatus: saprophyte or pathogen? Curr Opin Microbiol. 2005;8(4):385-392.

4. Lin SJ, Schranz J, Teutsch SM. Aspergillosis casefatality rate: systematic review of the literature. Clin Infect Dis. 2001;32(3):358-366.

5. Damek DM, Lillehei KO, Kleinschmidt-DeMasters
BK. Aspergillus terreus brain abscess mimicking tumor progression in a patient with treated glioblastoma multiforme. Clin Neuropathol. 2008;27(6):400-407.

6. Nadkarni T, Goel A. Aspergilloma of the brain: an overview. J Postgrad Med. 2005;51 Suppl 1:S37-S41.

7. Rogers TR. Treatment of zygomycosis: current and new options. J Antimicrob Chemother. 2008;61 Suppl 1:i35-i40.

8. Ahmadzai H, Raley DA, Masters L, Davies M. An unusual case of a pituitary fossa aspergilloma in an immunocompetent patient mimicking infiltrative tumour. J Surg Case Rep. 2013;2013(4).

9. Sood S, Sharma R, Gupta S, Pathak D, Rishi S. Neuroaspergillosis in an immunocompetent patient. Indian J Med Microbiol. 2007;25(1):67-69.

10. Phuttharak W, Hesselink JR, Wixom C. MR features of cerebral aspergillosis in an immunocompetent patient: correlation with histology and elemental analysis. AJNR Am J Neuroradiol. 2005;26(4):835-838

11. Tripathy SR, Mishra SS, Deo RC, Mohanta I, Tripathy K. Aqueductal Stenosis Has a New DimensionAspergillosis: A Rare Case Report and Review of the Literature. World Neurosurg. 2015;84(5):1494-1496.

12. Saghrouni F, Ben YY, Gheith S, Bouabid Z, Ben AJ, Khammari I, et al. Twenty-nine cases of invasive aspergillosis in neutropenic patients. Med Mal Infect. 2011;41(12):657-662.

13. Iplikcioglu AC, Bek S, Bikmaz K, Ceylan D, Gokduman CA. Aspergillus pituitary abscess. Acta Neurochir (Wien). 2004;146(5):521-524.

14. Liu W, Chen H, Cai B, Li G, You C, Li H. Successful treatment of sellar aspergillus abscess. J Clin Neurosci. 2010;17(12):1587-1589.

15. Alrajhi AA, Enani M, Mahasin Z, Al-Omran K. Chronic invasive aspergillosis of the paranasal sinuses in immunocompetent hosts from Saudi Arabia. Am J Trop Med Hyg. 2001;65(1):83-86.

16. Yip CM, Hsu SS, Liao WC, Chen JY, Liu SH, Chen $\mathrm{CH}$. Orbital apex syndrome due to aspergillosis with subsequent fatal subarachnoid hemorrhage. Surg Neurol Int. 2012;3:124.

17. Miki Y, Tomiyama M, Haga R, Nishijima H, Suzuki C, Nishijima M, et al. Fatal subarachnoid hemorrhage caused by Aspergillus arteritis without angiographic abnormalities. Neuropathology. 2012;32(5):566-569.

18. Ho CL, Deruytter MJ. CNS aspergillosis with mycotic aneurysm, cerebral granuloma and infarction. Acta Neurochir (Wien). 2004;146(8):851-856.

19. Speth C, Rambach G, Lass-Florl C, Wurzner R, Gasque $\mathrm{P}$, Mohsenipour I, et al. Culture supernatants of patient- 
derived Aspergillus isolates have toxic and lytic activity towards neurons and glial cells. FEMS Immunol Med Microbiol. 2000;29(4):303-313.

20. Conant MM, Sha BE, Proia LA. Use of posaconazole delayed-release tablets for treatment of invasive aspergillosis. Mycoses. 2015;58(5):313-314.
21. Ozcelik T, Ozkalemkas F, Kocaeli H, Altundal Y, Ener B, Ali R, et al. Successful treatment of neuroaspergillosis in a patient with acute lymphoblastic leukemia: role of surgery, systemic antifungal therapy and intracavitary therapy. Mikrobiyol Bul. 2009;43(3):499-506. 